\title{
SECURE COMMUNICATION USING PRACTICAL SYNCHRONIZATION BETWEEN TWO DIFFERENT CHAOTIC SYSTEMS WITH UNCERTAINTIES
}

\author{
Jianping Cai *, Meili Lin, Zhengzhong Yuan \\ Department of Mathematics, Zhangzhou Normal University, Zhangzhou 363000, China \\ mathcai@hotmail.com
}

\begin{abstract}
Adaptive control technique is used to design suitable controllers to synchronize two different coupled chaotic systems with uncertainties. The information signal hidden in chaotic signal is transmitted and then successfully recovered at the receiver once synchronization is achieved. Different chaotic systems with uncertainties are chosen respectively as the transmitter and receiver systems to ensure higher security in communication. Numerical simulation examples verify the effectiveness of the proposed method.
\end{abstract}

Keywords- chaos synchronization, secure communication, adaptive control, different chaotic systems

\section{INTRODUCTION}

Chaos synchronization has been widely investigated for its advantages in practical applications, particularly in secure communication [1-3]. The process of secure communication via chaos synchronization is outlined as follows. Firstly, the information signal is added to a much stronger chaotic signal in order to hide the information in transmitter. Secondly, the generated chaotic signal containing the information is transmitted to receiver. Finally, the information signal can be recovered successfully if synchronization is achieved between the transmitter and receiver under certain conditions.

Chaotic systems can be used in signal transmission in various ways, for examples, driving-response scheme [4], adaptive synchronization strategy [5], parameter modulation [6], observer-based synchronization [7]. Adaptive control is an effective method to synchronize mostly systems with unknown parameters or disturbances, which can improve the security of information and be more suitable for communication [8-10]. However, most of the mentioned literatures were designed to synchronize two identical chaotic systems and recover single signal hidden in the transmitter. Studies on secure communication with several different signals and different chaotic structures between the transmitter and receiver are limited. Adaptive synchronization between different chaotic systems with unknown parameters was investigated $[11,12]$. However, the effects of noise and parameter mismatch were not taken into account, and the controller contains all the information appeared in the error dynamical system or the transmitter-receiver systems, which is hard to realize in practical application. 
In this paper adaptive control technique is used to design suitable controllers to synchronize different coupled chaotic systems with uncertainties, including unknown parameters, internal or external perturbations. Such transmitter-receiver systems have higher security. Because of the existence of uncertainties, the transmitter-receiver systems are usually difficult to achieve complete synchronization. So a concept of practical synchronization is introduced in this paper. Under some conditions, Lyapunov stability theory ensures that the transmitter-receiver chaotic systems can achieve practical synchronization, and the information signal hidden in the transmitted chaotic signal can be successfully recovered at the receiver. The designed controllers contain only feedback terms and partial nonlinear terms of the systems, and they are easy to implement in practice. The Lorenz system and Chen system are chosen as the illustrative example to verify the validity of the proposed method.

\section{SECURE COMMUNICATION SCHEME}

Consider a class of chaotic systems with unknown parameters and disturbance as the transmitter

$$
\left\{\begin{array}{l}
\dot{x}=f(x)+F(x) \alpha+d(x, t)+s \\
x_{t}=C x+s
\end{array}\right.
$$

where $x \in R^{n}$ is the state vector, $\alpha \in R^{m}$ is the unknown parameter vector, $f(x) \in R^{n \times 1}$ and $F(x) \in R^{n \times m}$ are known function matrices, $d(x, t) \in R^{n \times 1}$ is the uncertainties including parameter perturbation and external disturbance, $s \in R^{n}$ is the information signal vector which will be recovered at the receiver, $C \in R^{n \times n}$ is a known constant matrix and $x_{t} \in R^{n}$ is the output vector.

Let $\Omega_{x} \subset R^{n}$ be a bounded region containing the whole attractor of transmitter system (1) such that no trajectory of system (1) ever leaves it. This assumption is simply based on the bounded property of chaotic attractor. Also, let $M \subset R^{m}$ be the set of parameter under which system (1) is in a chaotic state.

The receiver system with a controller is constructed as follows

$$
\left\{\begin{array}{l}
\dot{y}=g(y)+G(y) \beta+u(t) \\
y_{r}=C y
\end{array}\right.
$$

where $y \in R^{n}, \beta \in R^{m_{1}}$ is the unknown parameter vector, $g(y) \in R^{n \times 1}$ and $G(y) \in R^{n \times m_{1}}$ are known function matrices, $u(t) \in R^{n}$ is the control input vector, $y_{r} \in R^{n}$ is the output vector. Let $\Omega_{y} \subset R^{n}$ be a bounded region containing the whole attractor of receiver system (2) with $u(t)=0$.

Due to the uncertainties in the transmitter system (1), the receiver system (2) is usually difficult to achieve complete synchronization with the transmitter system (1). Therefore a concept of practical synchronization, also called synchronization with uniform ultimate boundedness in other literatures, is introduced in this paper.

Definition 1 The transmitter-receiver systems (1) and (2) achieve practical synchronization if for any initial state $x(0) \in \Omega_{x}$ and $y(0) \in \Omega_{y}$, there exist constants 
$h>0$ and $T_{0}>0$ such that the trajectory $x(t, x(0))$ of system (1) and trajectory $y(t, y(0))$ of system (2) satisfy

$$
\|x(t, x(0))-y(t, y(0))\|<h \text {, for any } t>T_{0},
$$

where $\|$.$\| denotes the Euclidean norm, and h$ indicates the error bound.

According to this definition, our control objective is to design a suitable adaptive controller $u(t)$ such that the receiver system (2) eventually synchronizes with the transmitter system (1), and finally reconstruct the informal signal. To this end, some assumptions are given as follows.

Assumption 1 The disturbance vector $d(x, t)$ and information signal $s$ are norm bounded respectively, namely, $\|d(x, t)\| \leq L_{d},\|s(t)\| \leq L_{s}$, where $L_{d}$ and $L_{s}$ are positive constants.

Assumption 2 The function vectors $f(\cdot)$ and $g(\cdot)$ are continuous on a bounded closed region $\Omega$ containing both $\Omega_{x}$ and $\Omega_{y}$. So there exists a positive constant $L_{f}$ such that

$$
\|f(x)-g(x)\| \leq L_{f}, x \in \Omega \text {. }
$$

Assumption 3 The function vector $g(\cdot)$ satisfies the Lipschitz condition, that is, there exists a positive constant $L_{g}$ such that

$$
\|g(x)-g(y)\| \leq L_{g}\|x-y\|, \text { for any } x, y \in R^{n} .
$$

Theorem 1 The transmitter-receiver systems (1) and (2) can achieve practical synchronization and the informal signal $s$ can be recovered by

$$
\tilde{s}=x_{t}-y_{r},
$$

if the Assumptions 1-3 hold, and the controller $u(t)$ is designed as

$$
u(t)=F(x) \hat{\alpha}-G(y) \hat{\beta}+k e,
$$

where $e=x-y$ is the error variable, the feedback coefficient $k$ is a constant to be determined, and the adaptive variables $\hat{\alpha}$ and $\hat{\beta}$ satisfy the following adaptation laws

$$
\dot{\hat{\alpha}}=F(x)^{T} e, \dot{\hat{\beta}}=-G(y)^{T} e .
$$

Proof The error dynamical system is

$$
\dot{e}=\dot{x}-\dot{y}=f(x)-g(y)+F(x)(\alpha-\hat{\alpha})-G(y)(\beta-\hat{\beta})-k e+d(x, t)+s .
$$

Construct a Lyapunov function

$$
V=\frac{1}{2}\left[e^{T} e+(\alpha-\hat{\alpha})^{T}(\alpha-\hat{\alpha})+(\beta-\hat{\beta})^{T}(\beta-\hat{\beta})\right] .
$$

Using Assumptions 1-3 and Eqs.(4)-(6), the time derivative of $V$ satisfies,

$$
\begin{aligned}
\dot{V}= & e^{T} \dot{e}-(\alpha-\hat{\alpha})^{T} \dot{\hat{\alpha}}-(\beta-\hat{\beta})^{T} \dot{\hat{\beta}} \\
= & e^{T}(f(x)-g(y)+F(x)(\alpha-\hat{\alpha})-G(y)(\beta-\hat{\beta})-k e+d(x, t)+s) \\
& \quad-(\alpha-\hat{\alpha})^{T} F(x)^{T} e-(\beta-\hat{\beta})^{T}\left(-G(y)^{T} e\right) \\
= & e^{T}(f(x)-g(x))+e^{T}(g(x)-g(y))-k\|e\|^{2}+e^{T}(d(x, t)+s)
\end{aligned}
$$




$$
\begin{aligned}
& \leq L_{f}\|e\|+L_{g}\|e\|^{2}-k\|e\|^{2}+\left(L_{d}+L_{s}\right)\|e\| \\
& \leq \frac{\|e\|^{2}}{2 \varepsilon_{1}}+\frac{\varepsilon_{1} L_{f}^{2}}{2}+L_{g}\|e\|^{2}-k\|e\|^{2}+\frac{\|e\|^{2}}{2 \varepsilon_{2}}+\frac{\varepsilon_{2}\left(L_{d}+L_{s}\right)^{2}}{2} \\
& =\left(\frac{1}{2 \varepsilon_{1}}+\frac{1}{2 \varepsilon_{2}}+L_{g}-k\right)\|e\|^{2}+\frac{1}{2}\left(\varepsilon_{1} L_{f}^{2}+\varepsilon_{2}\left(L_{d}+L_{s}\right)^{2}\right)
\end{aligned}
$$

where $\varepsilon_{1}, \varepsilon_{2}$ are small positive constants, and the equality $a b \leq \frac{a^{2}}{2 c}+\frac{c b^{2}}{2}$ has been used, in which $c$ is a positive constant. Let

$$
\varepsilon=\frac{1}{2}\left(\varepsilon_{1} L_{f}^{2}+\varepsilon_{2}\left(L_{d}+L_{s}\right)^{2}\right) .
$$

If the feedback coefficient $k$ satisfies

$$
k \geq \frac{1}{2 \varepsilon_{1}}+\frac{1}{2 \varepsilon_{2}}+L_{g}+1
$$

then we have

$$
\dot{V} \leq-\|e\|^{2}+\varepsilon \text {. }
$$

By the inequality (9), we conclude that the trajectory of state error will approach to a hyper-ball determined by $\|e\| \leq \sqrt{\varepsilon}$, so practical synchronization can be achieved. From Eqs.(7)-(9), it is clear that $\varepsilon$ will be sufficiently small if $\varepsilon_{1}$ and $\varepsilon_{2}$ are chosen sufficiently small, which implies the synchronization error will also be sufficiently small. This can be realized simply by choosing larger value of $k$. Then the informal signal $s$ can be reconstructed approximately by

$$
\tilde{s}=x_{t}-y_{r}=C x+s-C y=s+C e .
$$

Remark The controller (4) contains only the feedback term and partial nonlinear terms of the systems, while the controllers in Refs.[12-17] include all the information appeared in the error dynamical system or the transmitter-receiver systems.

\section{ILLUSTRATIVE EXAMPLE}

Secure communication between Lorenz system and Chen system is presented to simulate the proposed method. The Lorenz system with unknown parameters and perturbations is chosen as the transmitter system

$$
\left\{\begin{array}{l}
\left(\begin{array}{l}
\dot{x}_{1} \\
\dot{x}_{2} \\
\dot{x}_{3}
\end{array}\right)=\left(\begin{array}{c}
0 \\
-x_{2}-x_{1} x_{3} \\
x_{1} x_{2}
\end{array}\right)+\left(\begin{array}{ccc}
x_{2}-x_{1} & 0 & 0 \\
0 & x_{1} & 0 \\
0 & 0 & -x_{3}
\end{array}\right)\left(\begin{array}{l}
\alpha_{1} \\
\alpha_{2} \\
\alpha_{3}
\end{array}\right)+\left(\begin{array}{l}
d_{1}(x, t) \\
d_{2}(x, t) \\
d_{3}(x, t)
\end{array}\right)+\left(\begin{array}{l}
s_{1} \\
s_{2} \\
s_{3}
\end{array}\right) \\
\left(\begin{array}{l}
x_{1 t} \\
x_{2 t} \\
x_{3 t}
\end{array}\right)=C\left(\begin{array}{l}
x_{1} \\
x_{2} \\
x_{3}
\end{array}\right)+\left(\begin{array}{l}
s_{1} \\
s_{2} \\
s_{3}
\end{array}\right)
\end{array}\right.
$$

Compared with Eq.(1), the relative notations are

$$
x=\left(x_{1}, x_{2}, x_{3}\right)^{T}, f(x)=\left(0,-x_{2}-x_{1} x_{3}, x_{1} x_{2}\right)^{T}, \quad F(x)=\operatorname{diag}\left(x_{2}-x_{1}, x_{1},-x_{3}\right),
$$




$$
\alpha=\left(\alpha_{1}, \alpha_{2}, \alpha_{3}\right)^{T}, d(x, t)=\left(d_{1}(x, t), d_{2}(x, t), d_{3}(x, t)\right)^{T}, s=\left(s_{1}, s_{2}, s_{3}\right)^{T} .
$$

The Chen system with unknown parameters and controllers is selected as the receiver system

$$
\left\{\begin{array}{l}
\left(\begin{array}{l}
\dot{y}_{1} \\
\dot{y}_{2} \\
\dot{y}_{3}
\end{array}\right)=\left(\begin{array}{c}
0 \\
-y_{1} y_{3} \\
y_{1} y_{2}
\end{array}\right)+\left(\begin{array}{ccc}
y_{2}-y_{1} & 0 & 0 \\
-y_{1} & y_{1}+y_{2} & 0 \\
0 & 0 & -y_{3}
\end{array}\right)\left(\begin{array}{l}
\beta_{1} \\
\beta_{2} \\
\beta_{3}
\end{array}\right)+\left(\begin{array}{l}
u_{1}(t) \\
u_{2}(t) \\
u_{3}(t)
\end{array}\right) \\
\left(\begin{array}{l}
y_{1 r} \\
y_{2 r} \\
y_{3 r}
\end{array}\right)=C\left(\begin{array}{l}
y_{1} \\
y_{2} \\
y_{3}
\end{array}\right)
\end{array}\right.
$$

Compared with Eq.(2), the relative notations are

$$
y=\left(y_{1}, y_{2}, y_{3}\right)^{T}, g(y)=\left(0,-y_{1} y_{3}, y_{1} y_{2}\right)^{T}, G(y)=\left(\begin{array}{ccc}
y_{2}-y_{1} & 0 & 0 \\
-y_{1} & y_{1}+y_{2} & 0 \\
0 & 0 & -y_{3}
\end{array}\right),
$$

$\beta=\left(\beta_{1}, \beta_{2}, \beta_{3}\right)^{T} . u(t)=\left(u_{1}(t), u_{2}(t), u_{3}(t)\right)^{T}$ is determined by Eqs.(4) and (5).

It is easy to verify that the Lorenz system and Chen system satisfy Assumptions 1-3.

Case 1 No disturbance is applied to the transmitter system, that is, $d(x, t)=0$. Suppose that the transmitted signal is $\left(s_{1}, s_{2}, s_{3}\right)^{T}=(0.2 \sin 2 t, 0.1 \operatorname{sign}(\sin (\pi t / 4)), 0)^{T}$, and

$$
C=\left(\begin{array}{ccc}
1 & -1 & 0 \\
0 & 1 & -1 \\
-1 & 0 & 1
\end{array}\right) .
$$

The Lorenz system with signal loading is in a state of chaos, whose attractor is displayed in Fig.1. Under the designed controller, the receiver system (11) can achieve practical synchronization with the transmitter system (10) as shown in Fig.2. It is clearly seen in Figs. 3 and 4 that the reconstructed signal $\tilde{s}$ coincides with the informal signal $s$ with good accuracy. The error between the transmitted and recovered signals can be reduced to desired accuracy by simply increasing the feedback coefficient $k$. In the simulation, the unknown parameters in systems (10) and (11) are assumed to be "known" as $\alpha=(10,28,8 / 3)^{T}$ and $\beta=(35,28,3)^{T}$. The feedback coefficient $k=50$, and the initial values are chosen arbitrarily as $x(0)=(1.4,-1,2.4)^{T}, y(0)=(-0.5,1.3,0.8)^{T}$, $\hat{\alpha}(0)=(1,4,-1)^{T}, \hat{\beta}(0)=(-2,2.1,1.4)^{T}$. 


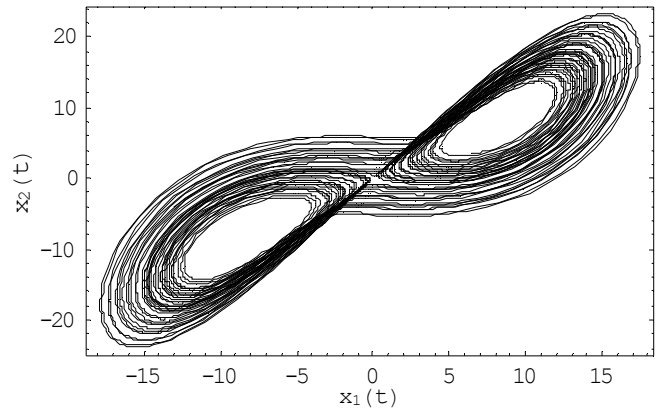

(a) $x_{1}-x_{2}$ plane

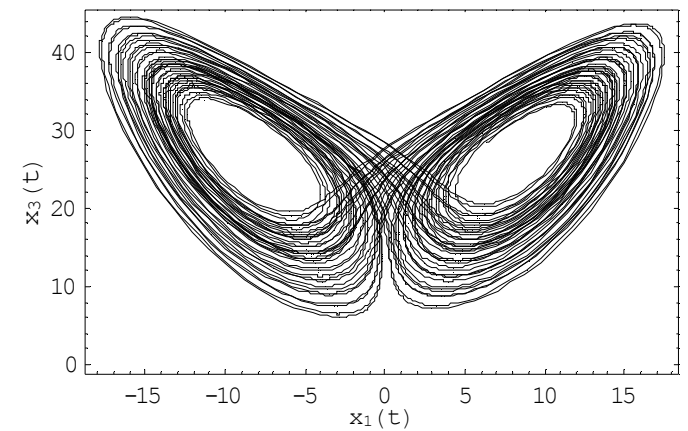

(b) $x_{1}-x_{3}$ plane

Fig. 1 Chaotic attractor of the Lorenz system with information signal loading

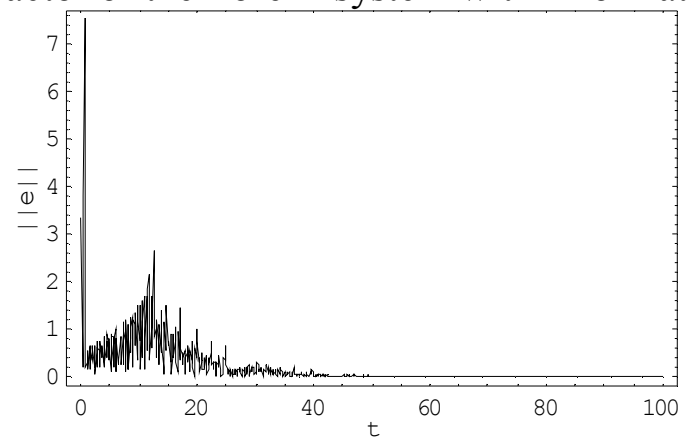

Fig.2 Synchronization error between systems (10) and (11)

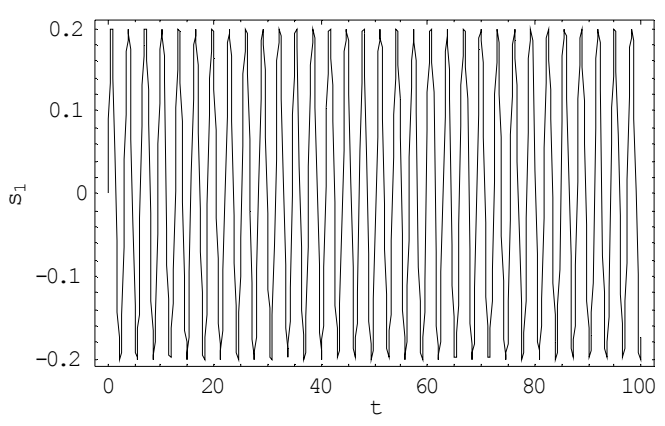

(a)Information signal $s_{1}$

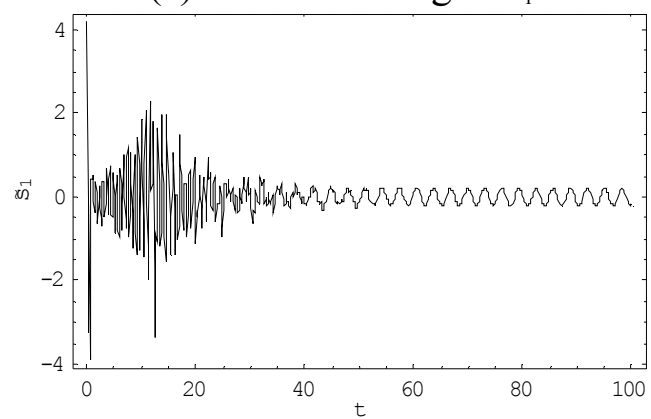

(c)Recovered signal $\tilde{s}_{1}$

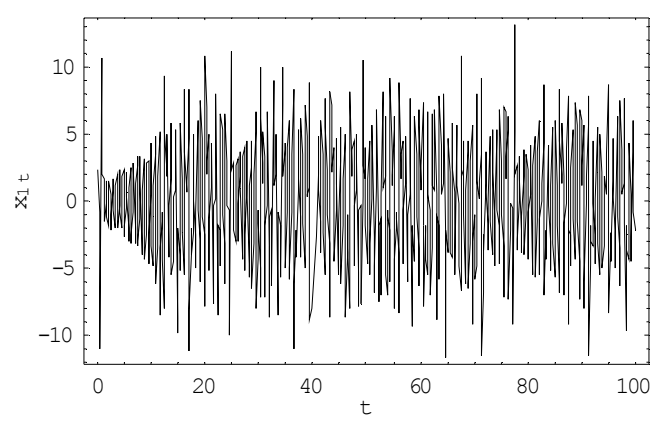

(b)Transmitted information $x_{1 t}$

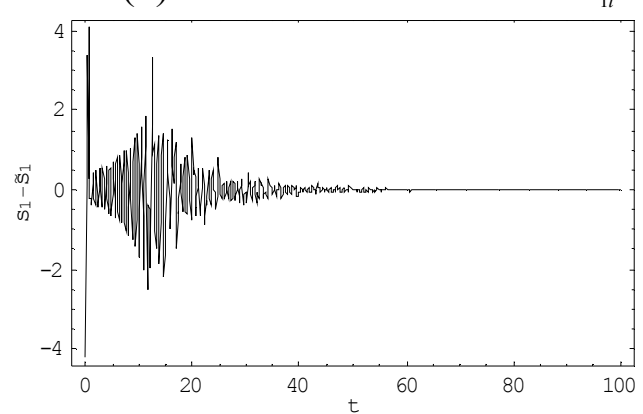

(d)Error signal $s_{1}-\tilde{s}_{1}$

Fig.3 Process of signal $s_{1}$ transmission and recovery without disturbance 


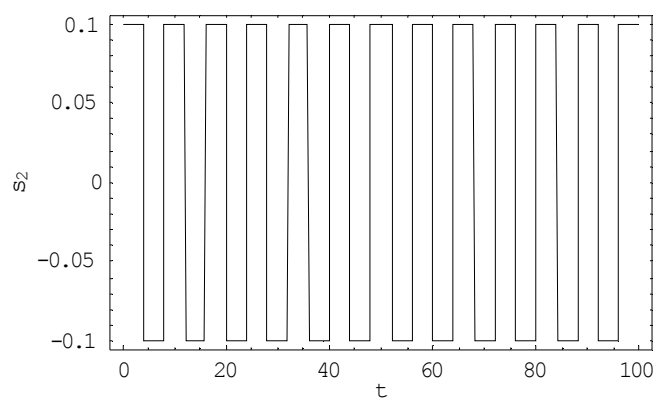

(a)Information signal $s_{2}$

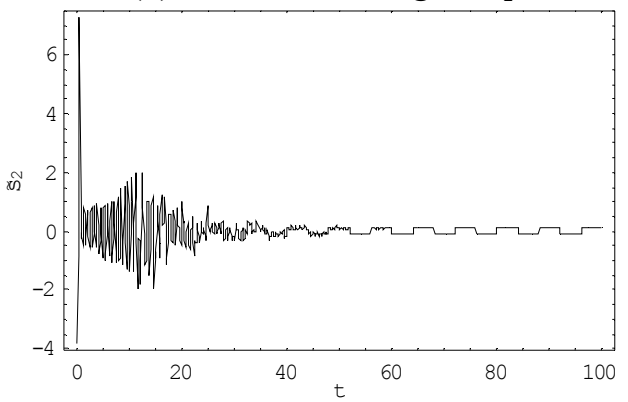

(c)Recovered signal $\tilde{s}_{2}$

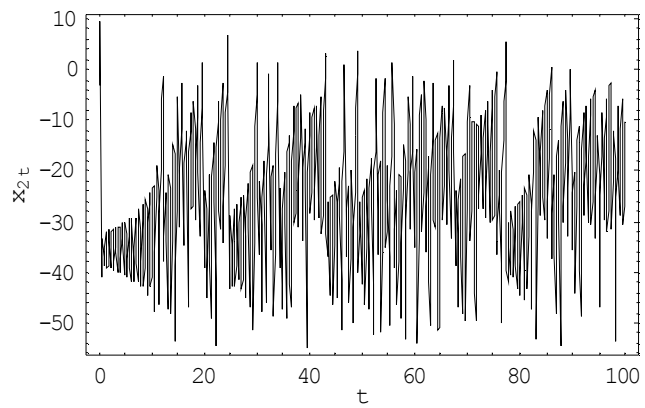

(b)Transmitted information $x_{2 t}$

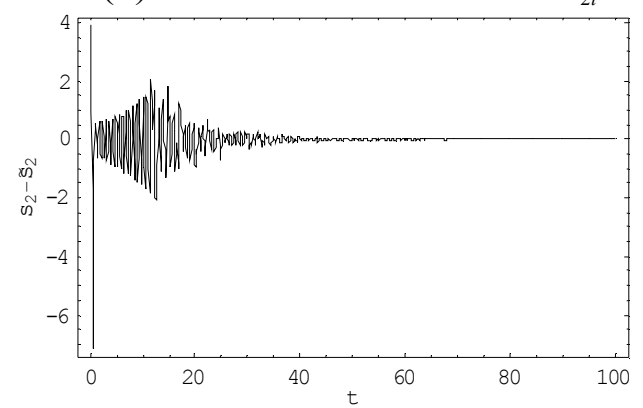

(d)Error signal $s_{2}-\tilde{s}_{2}$

Fig.4 Process of signal $s_{2}$ transmission and recovery without disturbance

Case 2 Parameter perturbation is applied to the transmitter system (10), for example,

$$
d(x, t)=\left(\lambda \sin (2 t) \cdot\left(10 x_{1}\right), \lambda \cos t \cdot x_{2}, \lambda \sin (3 t) \cdot\left(\frac{8}{3} x_{3}\right)\right)^{T} .
$$

Now the transmitter system (10) becomes,

$$
\begin{aligned}
& \dot{x}_{1}=10 x_{2}-10(1-\lambda \sin 2 t) x_{1}+0.2 \sin 2 t, \\
& \dot{x}_{2}=28 x_{1}-(1-\lambda \cos t) x_{2}-x_{1} x_{3}+0.1 \operatorname{sign}(\sin (\pi t / 4)), \\
& \dot{x}_{3}=x_{1} x_{2}-\frac{8}{3}(1-\lambda \sin 3 t) x_{3},
\end{aligned}
$$

where $\lambda$ represents the perturbed strength compared with the magnitude of state variables $x_{i}(i=1,2,3)$. The informal signals also can be well recovered. The results are shown in Figs. 5 and 6 with the perturbed strength $\lambda=1 \%$. The other values of parameters are the same as those of Case 1 . The above simulations are performed in computer algebraic system Mathematica, where instruction NDSolve is used.

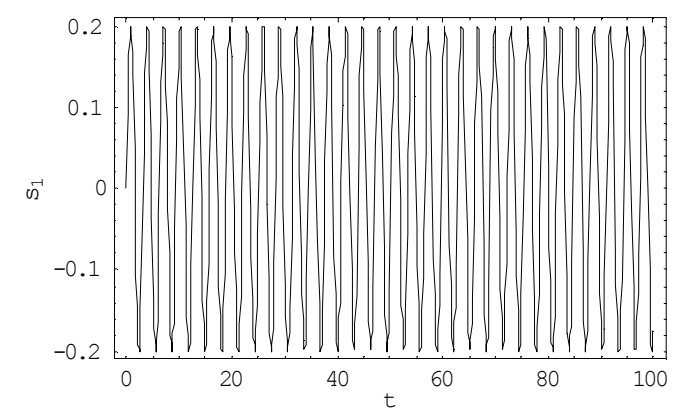

(a)Information signal $s_{1}$

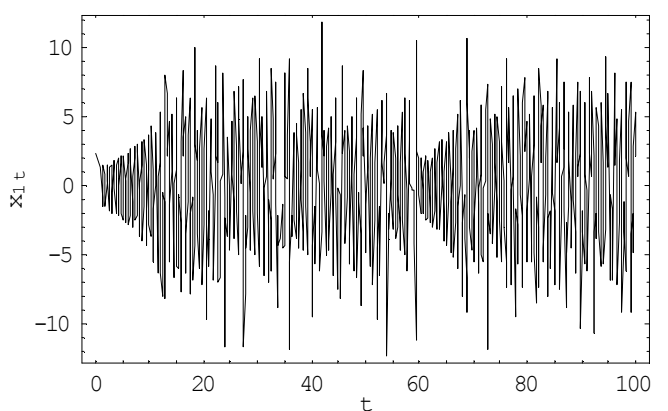

(b)Transmitted information $x_{1 t}$ 


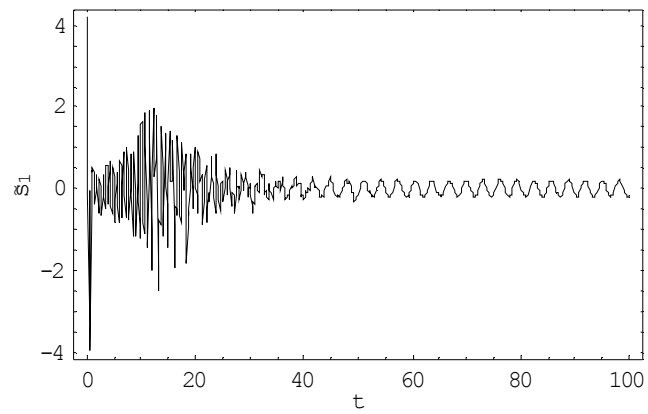

(c)Recovered signal $\tilde{s}_{1}$

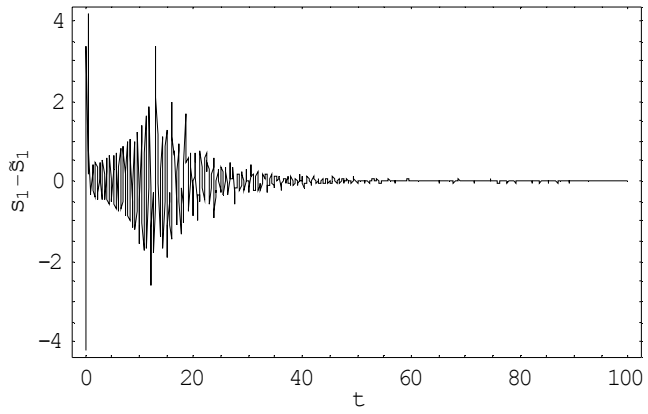

(d)Error signal $s_{1}-\tilde{s}_{1}$

Fig.5 Process of signal $s_{1}$ transmission and recovery under disturbances

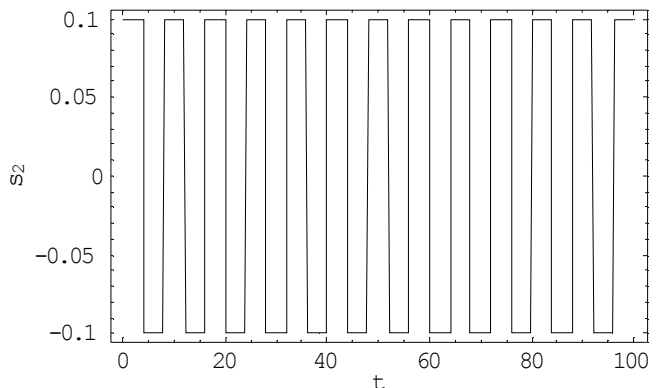

(a)Information signal $s_{2}$

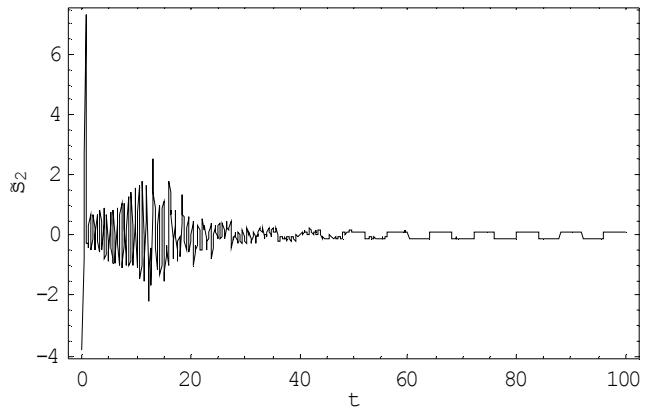

(c)Recovered signal $\tilde{s}_{2}$

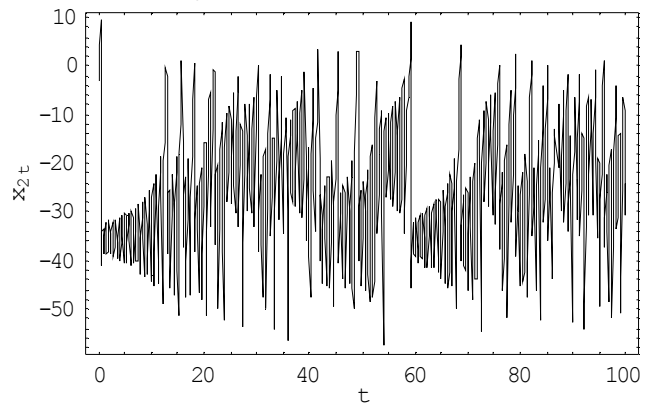

(b)Transmitted information $x_{2 t}$

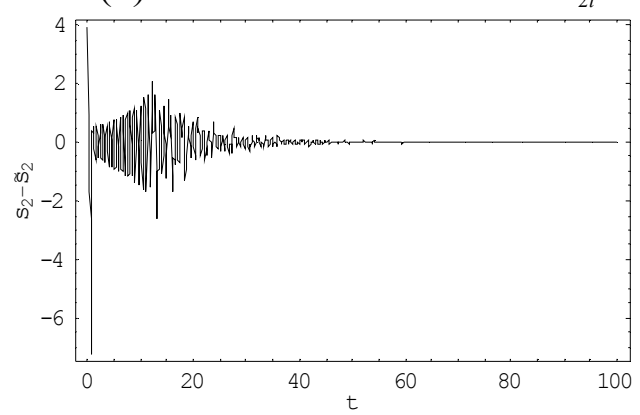

(d)Error signal $s_{2}-\tilde{s}_{2}$

Fig.6 Process of signal $s_{2}$ transmission and recovery under disturbances

Now we discuss the effect of disturbance on the error between the transmitted signal and recovered one. The enlarged figure of Fig.3(d) combined with Fig.5(d) at $t \in[50,100]$ is shown in Fig.7(a), and that of Fig.4(d) combined with Fig.6(d) is shown in Fig.7(b). We see clearly that the disturbances result in larger error between the transmitted and recovered signals. 


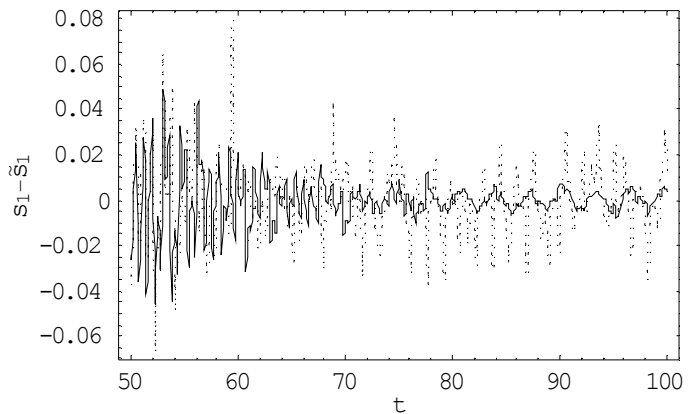

(a) Block curve for $s_{1}-\tilde{s}_{1}$ without disturbance and dashing curve for $s_{1}-\tilde{s}_{1}$ under disturbances

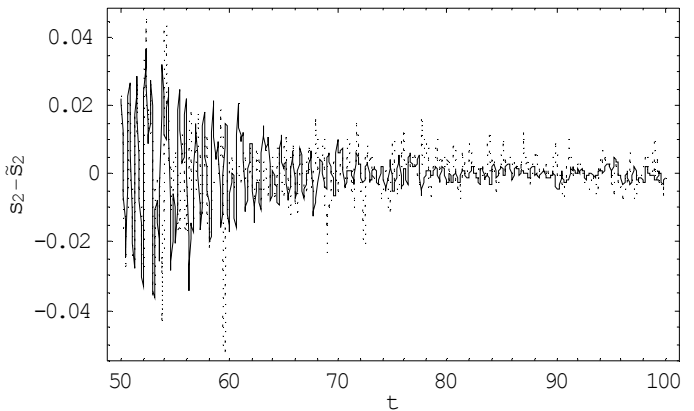

(b) Block curve for $s_{2}-\tilde{s}_{2}$ without disturbance and dashing curve for $s_{2}-\tilde{s}_{2}$ under disturbances

Fig.7 Effect of disturbance on the error between the transmitted and recovered signals

\section{CONCLUSIONS}

In this paper we design adaptive controllers to synchronize two different chaotic systems with uncertainties, containing unknown parameters, internal or external perturbations. Using Lyapunov stability theory, we prove that under some conditions the transmitter-receiver systems can achieve practical synchronization, and the information signal hidden in the transmitted chaotic signal can be successfully recovered at the receiver. Furthermore the error between the transmitted and recovered signals can be reduced to desired accuracy. The designed controllers contain only feedback terms and partial nonlinear terms of the systems, and they are easy to implement in practice. The Lorenz system and Chen system are chosen as the illustrative example to verify the validity of the proposed method.

Acknowledgement- Research is supported by the National Natural Science Foundation of China under grant No.60674049.

\section{REFERENCES}

1. L.M. Pecora and T.L. Carroll, Synchronization in chaotic systems, Physical Review Letters, 64, 821-824, 1990.

2. S. Boccaletti, J. Kurths , G. Osipov, D.L. Valladares and C.S. Zhou, The synchronization of chaotic systems, Physics Reports, 366, 1-101, 2002.

3. T. Yang, A Survey of chaotic secure communication systems, International Journal of Computational Cognition, 2(2), 81-130, 2004.

4. L.O. Chua and C.W. Wu, A simple way to synchronize chaotic systems with applications to secure communication systems, International Journal of Bifurcation and Chaos, 3( 6), 1619-1627, 1993.

5. F. Tang, An adaptive synchronization strategy based on active control for demodulating message hidden in chaotic signals, Chaos, Solitons and Fractals, 37, 1090-1096, 
6. S. Bowong, F.M.M. Kakmeni and M.S. Siewe, Secure communication via parameter modulation in a class of chaotic systems, Communications in Nonlinear Science and Numerical Simulation, 12, 397-410, 2007.

7. F.L. Zhu, Observer-based synchronization of uncertain chaotic system and its application to secure communications, Chaos, Solitons and Fractals, doi:10.1016/j.chaos.2007.10.052, 2008.

8. T.L. Liao and S.H. Tsai, Adaptive synchronization of chaotic systems and its application to secure communications, Chaos, Solitons and Fractals, 11(9), 13871396, 2000.

9. M. Feki, An adaptive chaos synchronization scheme applied to secure communication, Chaos, Solitons and Fractals, 18(1), 141-148, 2003.

10. Y.H. Sun, J.D. Cao and G. Feng, An adaptive chaotic secure communication scheme with channel noises, Physics Letters A, 372, 5442-5447, 2008.

11. S. Li, W. Xu and R.H. Li, Synchronization of two different chaotic systems with unknown parameters, Physics Letters A, 361, 98-102, 2007.

12. H.G. Zhang, W. Huang, Z.L. Wang and T.Y. Chai, Adaptive synchronization between different chaotic systems with unknown parameters, Physics Letters A, 350, 363-366, 2006.

13. X.Y. Chen and J.F. Lu, Adaptive synchronization of different chaotic systems with fully unknown parameters, Physics Letters A, 364, 123-128, 2007.

14. J. Huang, Adaptive synchronization between different hyperchaotic systems with fully uncertain parameters, Physics Letters A, 372, 4799-4804, 2008.

15. W. Xu, L.X. Yang and Z.K. Sun, Full- and reduced-order synchronization of a class of time-varying systems containing uncertainties, Nonlinear Dynamics, 52, 19-25, 2008.

16. X.Y. Wang and M.J. Wu, Adaptive robust synchronization for a class of different uncertain chaotic systems, International Journal of Modern Physics B, 22(23), 4069-4082, 2008.

17. X.C. Li, W. Xu and Y.Z. Xiao, Adaptive tracking control of a class of uncertain chaotic systems in the presence of random perturbations, Journal of Sound and Vibration, 314, 526-535, 2008. 